\title{
A Rare Cause of Hemoptysis in Patients with Behcet's Disease: Hughes-stovin Syndrome: About One Case
}

\author{
Amine EI Masloumi*, Jean Frederic Adjimabou, Anass Chehboun, Badr Boutakioute, \\ Meriem Ouali Idrissi, Najat Cherif Idrissi El Ganouni \\ Radiology Department, Arrazy Hospital, Mohammed VIth Teaching Center, Cadi Ayad University, Marrakesh, Morocco

\section{Email address:} \\ a.elmasloumi@gmail.com (A. El Masloumi),jfredericadjimabou@yahoo.fr(J. F. Adjimabou), \\ chehboun.anass@gmail.com (A. Chehboun),badrboutakioute@gmail.com (B. Boutakioute), m.oualidrissi@gmail.com (M. O. Idrissi), \\ cherifidrissiganouni@gmail.com (N. C. I. El Ganouni) \\ ${ }^{*}$ Corresponding author
}

\section{To cite this article:}

Amine El Masloumi, Jean Frederic Adjimabou, Anass Chehboun, Badr Boutakioute, Meriem Ouali Idrissi, Najat Cherif Idrissi El Ganouni. A Rare Cause of Hemoptysis in Patients with Behcet's Disease: Hughes-stovin Syndrome: About One Case. International Journal of Medical Imaging. Vol. 8, No. 1, 2020, pp. 16-19. doi: 10.11648/j.jimi.20200801.13

Received: February 18, 2020; Accepted: February 28, 2020; Published: March 10, 2020

\begin{abstract}
Hughes-Stovin Syndrome (HSS) is a very rare condition characterized by pulmonary aneurysms and peripheral thrombophlebitis of lower limbs. The pathogenesis of Hughes Stovin Syndrome is unknown. It's considered as a clinical variant of Behçet's disease. The main clinical manifestation is haemoptysis and dyspnea. The management of HSS can either be medical or surgical. We report the case of a young patient of 25 years old followed for Bechet's disease, who presents for a medium abundance hemoptysis with a swelling of the lower limbs evolving for 20 days, the radiological investigations made it possible to pose the diagnosis of the Syndrome of Huges-Stovin showing thrombosed aneurysms of the pulmonary arteries with thrombophlebitis of the lower limbs, the patient benefited from an immunosuppressive treatment, the evolution was marked by a good clinical evolution.
\end{abstract}

Keywords: Behcet, Pulmonary Aneurysms, Thrombophlebitis, Hugues-stovin

\section{Introduction}

Hughes-Stovin syndrome is a very rare condition characterized by thrombophlebitis and multiple pulmonary and / or bronchial aneurysms. So far, less than 40 published cases have been described in the English medical literature. The exact etiology and pathogenesis are unknown. Possible causes include infections and angiodysplasia. This syndrome has also been considered a variant of Behçet's disease. Medical management includes the use of steroids and cytotoxic agents. Cyclophosphamide, in particular, is a therapeutic of choice. Antibiotics have no proven role, while anticoagulants and thrombolytic agents are generally contraindicated due to an increased risk of fatal hemorrhage.

\section{Observation}

We report the case of a 25 years old man followed for Behçet's disease for two years on colchicine and cortancyl, who stopped taking immurel for one year without medical advice, who was admitted in the emergency room for a medium abundance hemoptysis evolving for two months with recent appearance 20 days ago of a swelling of the lower limbs evolving in a context of febrile sensations and preservation of the general state. The clinical examination assessed a patient who was conscious, hemodynamically and respiratory stable. On the other hand, the examination of the lower limbs assessed a loss of physiological sloshing of the calves more marked on the right side with a positive Homans sign. A venous Doppler ultrasound was performed revealing a deep and extensive venous thrombosis of the two lower limbs (Figures 1 and 2). 

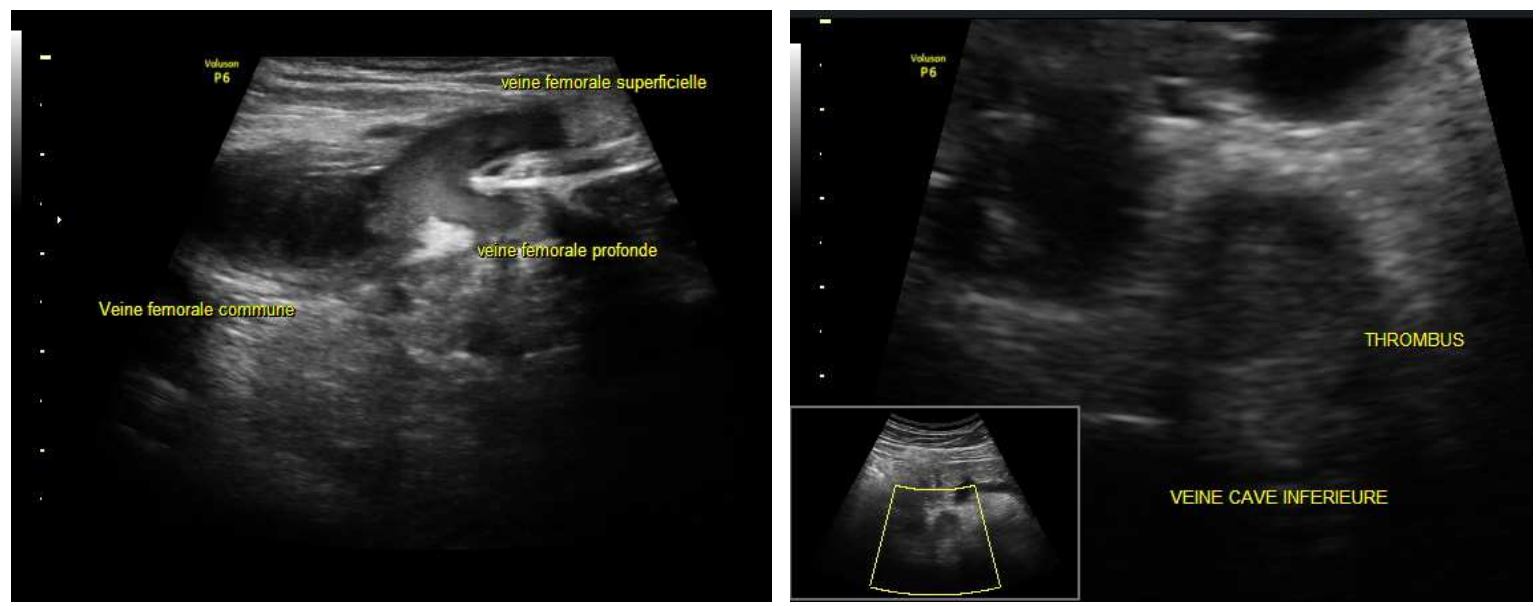

Figure 1. Lower limbs ultrasound B mode: Thrombosis of the venous network of the right lower limb (superficial, deep and common femoral veins extended to the inferior vena cava).

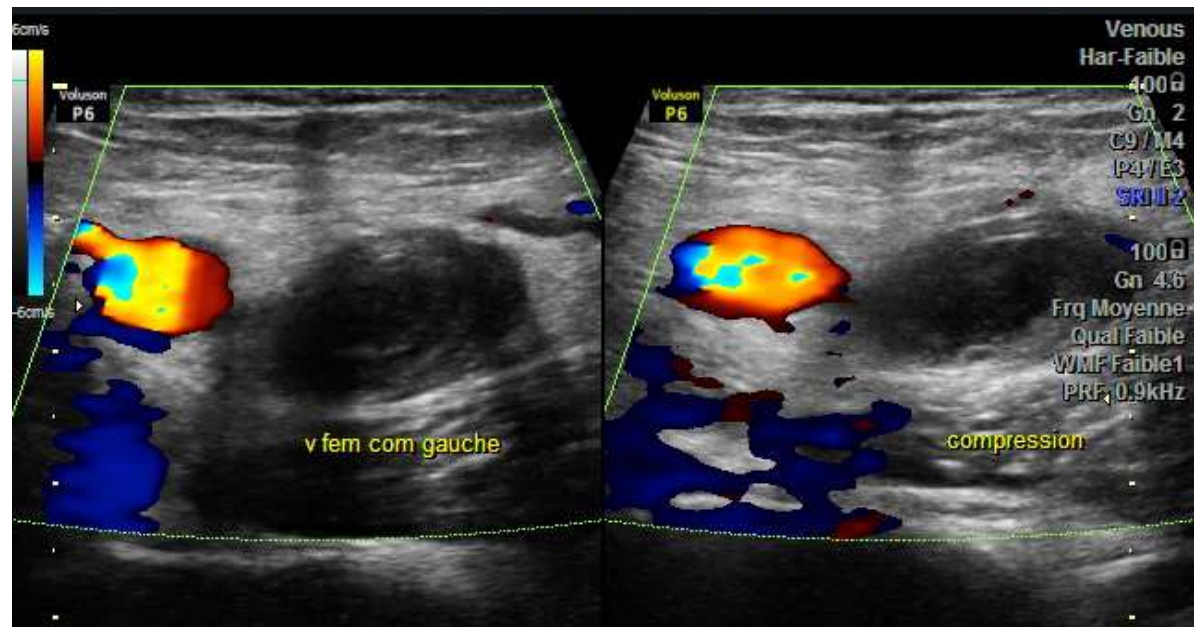

Figure 2. Lower limbs ultrasound color mode: Thrombosis of the left femoral vein, that is enlarged and non compressible.

Considering The association of a deep venous thrombosis and medium abundance hemoptysis, a chest $\mathrm{CT}$ angioscan was performed to rule out pulmonary embolism showing an aneurysmal dilation of the right upper ventral segmental artery, measuring $14.5 \mathrm{~mm}$ in diameter extended by $15 \mathrm{~mm}$, seat of hypodense endoluminal thrombus (Figure 3); aneurysmal dilation of the right medial-lower segmental artery, measuring $12 \mathrm{~mm}$ in diameter extended by $20 \mathrm{~mm}$, site of an endoluminal thrombus (Figure 4) and aneurysmal dilation of the right latero-lower segmental artery, measuring $15 \mathrm{~mm} 18 \mathrm{~mm}$ in diameter, site of an endoluminal thrombus.

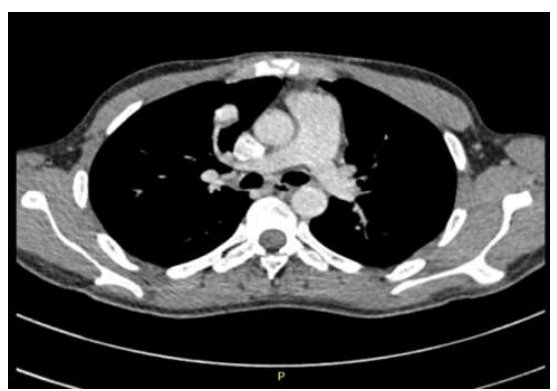

Figure 3. Aneurysmal dilation of the right upper ventral segmental artery, site of a thrombus.



Figure 4. Aneurysmal dilation of the right latero-inferior segmental artery that contains a thrombus.

The cardiac ultrasound did not reveal any abnormality. The physical examination also showed an oral aphthosis. The assessment of thrombophilia with assay of the circulating anticoagulant as well as anti-phospholipid antibodies was negative. There was an inflammatory syndrome with a CRP at $58 \mathrm{mg} / \mathrm{L}$ and hyperfibrinogenemia at $6 \mathrm{~g} / \mathrm{L}$. Infectious specimens, including blood cultures, syphilis serology, and repeated echocardiograms, excluded infectious endocarditis. The patient had received a bolus of methylprednisolone $(1.5 \mathrm{~g}$ / day for 3 days) and intravenous cyclophosphamide cycle (6 
cycles of $1 \mathrm{~g}$ at a monthly rate). Under this treatment and with 7 months of follow-up, the patient no longer had thromboembolic recurrences and remained perfectly asymptomatic. The diagnosis retained was the Hughes-Stovin syndrome.

\section{Discussion}

\subsection{History}

Hughes-Stovin Syndrome was named by two British doctors, Drs. John Patterson Hughes and Peter George Ingle Stovin. They first described the results of the syndrome (deep venous thrombosis syndrome and segmental pulmonary aneurysms), in a total of four patients with pulmonary artery aneurysms in 1959 [1, 2].

\subsection{Epidemiology}

It is an extremely rare disease, with less than 40 cases published in the English medical literature. It generally affects the young population with a range of ages between 12 and 48 years old with a strong male predilection [3-4]. HSS does not seem to have a preponderance for any geographic location. Huges Stovin syndrome has been reported from various geographic areas, including North America, Europe, Africa and Asia [5-6]. None of the reports have mentioned consanguineous marriages in the parents of patients suffering from SHS, thus, the genetic basis and familial predisposition of HSS remains unclear.

\subsection{The Clinic}

There are no diagnostic criteria or pathognomonic signs for this syndrome. Generally, the syndrome is characterized by signs of thrombophlebitis associated with multiple pulmonary and / or bronchial aneurysms [7-8]. Behcet's disease (BD), is also associated with this aneurysm-thrombosis combination. The distribution of the vascular component of the syndrome is as follows: arterial (7\%), venous (25\%) or both (68\%) [ 9]. The clinical paradigm of HSS can be divided into three phases $[8,4]$ : first the Symptoms of thrombophlebitis then the formation of pulmonary and / or bronchial vascular aneurysms, then the aneurysmal rupture leading to massive hemoptysis and death It may be similar to the Behcet disease model with interaction of multiple genetic, environmental, immunological and endothelial factors is most likely to be involved in BD pathogenesis [10]. However, some authors consider Hughes-Stovin syndrome as an incomplete form of Behcet's disease, due to the similarity between the two in terms of clinical, radiological and pathological results (11-12). Therefore, Behcet's disease, which commonly affects young men, is considered the primary differential diagnosis (13). The major and mandatory criterion for the diagnosis of Behcet's disease are oral ulcers that recur at least three times a year, which should be accompanied by at least two of the minor criteria (not necessarily simultaneously), including recurrent genital ulcers, eye and skin lesions. Other causes of pulmonary artery aneurysms are trauma, infections, pulmonary hypertension and Marfan syndrome. (12-13).

\subsection{Imagery}

Doppler ultrasound of the lower limbs is an important part of the diagnosis and should therefore be performed to assess the presence of deep vein thrombosis on the basis of reasonable clinical suspicion. The diagnosis of venous thrombosis is based on the incompressible nature of the venous thrombosis segments and for the segments not accessible for the compression study (iliac veins) on the absence of Doppler flow [14].

Chest $\mathrm{X}$ rays illustrate aneurysms of the arteries by showing hilar enlargements or round, lobulated opacities [15].

The chest angioscan can demonstrate high quality vascular images with a minimal amount of contrast material. Helical computed tomography angiography currently offers greater accuracy for visualisation of large systemic arteries than conventional angiography. It can be considered an emerging and effective standard in the diagnosis of pulmonary artery aneurysms due to its non-invasive nature, ease of execution and increasing availability. It allows to visualize the lumen of the vessel, the wall thrombus, the vascular wall and the mediastinum. [16, 17]

Magnetic resonance angiography (MRA) is relatively less efficient than CT in detecting small aneurysms [18, 19].

\subsection{Treatment}

Regarding the therapeutic modalities, there is no specific treatment for Hughes-Stovin syndrome; the most widely used treatment option is immunosuppressive therapy combining glucocorticoids and cyclophosphamide, which can potentially stabilize aneurysms or even promote their regression in some cases (12). The use of anticoagulants is controversial due to the risk of fatal hemoptysis, and administered only in certain cases and in conjunction with immunosuppressive therapy (11-12).

Interventional vascular radiology may offer an effective therapeutic option, but has not been studied in detail in this context.

Interventional radiologists should be familiar with the procedures used to treat various abnormalities of the pulmonary arteries. These well-established techniques, which avoid open surgery. Intra-arterial thrombolysis, percutaneous thrombectomy or fragmentation of the embolism may be performed in patients with potentially fatal pulmonary embolism [20]. Embolization with a steel coil catheter is an effective and secure method of preventing rupture of arterial aneurysms.

\section{Summary}

Hughes-Stovin syndrome should be evoked before any deep vein thrombosis occurring in a young man with Behçet's disease and, a fortiori when repeated hemoptysis occurs. In these conditions, chest angioscan must be performed systematically looking for aneurysmal images. In addition to medical treatment, arterial embolization is an essential 
therapeutic tool in the event of massive hemoptysis, which is life-threatening.

\section{References}

[1] Hughes JP, Stovin PG: Segmental pulmonary artery aneurysms with peripheral venous thrombosis. Br J Dis Chest 1959, 53: 19-27.

[2] Hughes-Stovin Syndrome Umair Khalid and Taimur Saleem Orphanet Journal of Rare Diseases 2011, 6: 15

[3] Weintraub JL, DeMayo R, Haskal ZJ, Susman J: SCVIR annual meeting film panel session: diagnosis and discussion of case 1 . J Vasc Interv Radiol 2001, 12: 531-4.

[4] Khalil A, Parrot A, Fartoukh M, Marsault C, Carette MF: Images in cardiovascular medicine. Large pulmonary artery aneurysm rupture in Hughes-Stovin syndrome: multidetector computed tomography pattern and endovascular treatment. Circulation 2006, 114: e380-1.

[5] Kim JT, Oh TY, Chang WH: Rare case of multiple pulmonary artery aneurysms with caval thrombosis-Hughes-Stovin syndrome. Eur J Cardiothorac Surg 2007, 31: 561-2.

[6] Chalazonitis AN, Lachanis SB, Mitseas P, Argyriou P, Tzovara J, Porfyrides P, Sotiropoulou E, Ptohis N: Hughes-Stovin Syndrome: a case report and review of the literature. Cases J 2009, 2: 98 .

[7] Farber HW, McDermott $\mathrm{S}$ et all Year-Old Woman with Recurrent Venous Thromboembolism and Pulmonary Artery Aneurysm. N Engl J Med. 2018 Apr 12; 378 (15): 1430-1438

[8] Reimold WV, Emmrich J, Harmjanz D, Kochsiek K: Multiple aneurysms of the pulmonary artery following recurrent septic pulmonary embolism (Hughes-Stovin syndrome): report of 1 case. Arch Klin Med 1968, 215: 1-18.

[9] Al-Jahdali H: Massive hemoptysis and deep venous thrombosis presenting in a woman with Hughes-Stovin syndrome: a case report. J Med Case Reports 2010, 4: 109.
[10] Kapsimali VD, Kanakis MA, Vaiopoulos GA, Kaklamanis PG: Etiopathogenesis of Behçet's disease with emphasis on the role of immunological aberrations. Clin Rheumatol 2010, 29: 1211-6.

[11] Cruz VA, Muniz YA, Silva Torres PPT, et al. Síndrome de Hughes-Stovin. Rev Bras Reumatol. 2009; 49: 747-52.

[12] Khalid U, Saleem T. Hughes-Stovin syndrome. Orphanet J Rare Dis. 2011; 6: 15.

[13] Fernandes GL, Teixeira AA, Antón AGS, et al. Churg-Strauss syndrome: a case report. Radiol Bras. 2014; 47: 259-61.

[14] M.-P. Revel-Dubois, J. de Laveaucoupet. Echo-Doppler des thromboses veineuses Journal de Radiologie Volume 85, Issue 9, September 2004, Page 1208

[15] Erkan F, Kiyan E, Tunaci A: Pulmonary complications of Behçet's disease. Clin Chest Med 2002, 23: 493-503.

[16] Balci NC, Semelka RC, Noone TC: Multiple pulmonary aneurysms secondary to Hughes-Stovin syndrome: demonstration by MRangiography. J Magn Reson Imaging 1998, 8: 1323-5.

[17] Ketchum ES, Zamanian RT, Fleischmann D: CT angiography of pulmonary artery aneurysms in Hughes-Stovin syndrome. AJR Am J Roentgenol 2005, 185: 330-2

[18] Herb S, Hetzel M, Hetzel J, Friedrich J, Weber J: An unusual case of Hughes-Stovin syndrome. Eur Respir J 1998, 11: 1191-3.

[19] Tsai CL, Lu TS, Tsai KC, Chen WJ: Hemoptysis ca used by Hughes-Stovin syndrome. Am J Emerg Med 2005, 23: 209-11.

[20] Voiriot G, Parrot A, Antoine $M$ et All. Transcatheter embolotherapy of pulmonary artery aneurysms as emergency treatment of hemoptysis in Behcet patients: experience of a referral center and a review of the literature. Internal and Emergency Medecine. 2018 Jun; 13 (4): 491-500.

[21] Pelage JP, El Hajjam M et All. Pulmonary artery interventions: an overview Radiographics. 2005 Nov-Dec. 